\title{
La relación entre la percepción subjetiva del funcionamiento cognitivo y el autoestigma con la experiencia de recuperación de las personas con enfermedad mental grave.
}

The relationship between self-reported cognitive functioning and self-stigma with the subjective recovery process in people with serious mental illness.

Mariasun Garay Arostegui a, Verónica Pousa Rodríguez ${ }^{\text {b }}$, Lucia Pérez Cabeza ${ }^{\mathrm{c}}$.

${ }^{a}$ Psicóloga clínica. Red de Salud Mental de Bizkaia. Osakidetza. España. ${ }^{b}$ Residente de Psicología Clínica. Hospital Universitario de Basurto. Osakidetza. Bilbao, España. ${ }^{C}$ Psiquiatra. Red de Salud Mental de Bizkaia. Osakidetza. España.

Correspondencia: Mariasun Garay Arostegui (mariaasuncion.garayarostegui@osakidetza.net)

Recibido: 10/09/2013; aceptado con modificaciones: 16/02/2014

RESUMEN: Se analizan las variables psicosociales intervinientes en el desarrollo del proceso de recuperación de las personas con trastorno mental grave focalizándose sobre aspectos subjetivos relacionados con la autopercepción.

Se diseña un estudio descriptivo de series de casos transversales. La muestra está formada por 59 usuarios de dos Hospitales de Día de Salud Mental a los que se les aplicaron diversas escalas objetivas y subjetivas con el objetivo de revisar la interrelación entorno a la experiencia de recuperación; así se valoró su vivencia de recuperación, autoestigma, el insight cognitivo y la metacognición sobre el funcionamiento cognitivo y social, teniendo en cuenta los aspecto objetivables tales como aspectos clínicos, funcionales y sociales generales.

Los resultados subrayan la importancia de factores perceptivos subjetivos y metacognitivos en el proceso personal de recuperación. Apuntan a la existencia de relaciones significativas entre el autoestigma y la recuperación. Se concluye que la sensación de estigma internalizado y la presencia de actividad psicótica redundan en una menor experiencia de recuperación. A su vez, las dificultades en las relaciones sociales y la percepción de quejas subjetivas sobre el funcionamiento cognitivo se asocian a una mayor sensación personal de autoestigma. Se considera la aplicación consecuente de estos hallazgos en el abordaje clínico.

PALABRAS CLAVE: Esquizofrenia, recuperación, autoestigma, metacognición, insight cognitivo.
ABSTRACT: In this study, we analyze the psychosocial variables involved in the development of the recovery process of people with severe mental disorders. This study aims to explore aspects related with subjective self-perception and the recovery process.

The study had a descriptive cross sectorial design. The sample consisted of 59 participants recruited from two mental health day hospitals. Several objective and subjective scales were applied in order to review their relationship around the recovery experience, and to evaluated their experience of recovery, self-stigma, cognitive insight and metacognition on cognitive and social functioning. We also took into account objectified aspects such as clinical, functional and general social situation.

The results underline the importance of subjective and metacognitive perceptual factors in the personal process of recovery. These results suggest the existence of a significant relationship between selfstigma and recovery. They conclude that the sense of internalized stigma and the presence of psychotic activity imply lower recovery experience. In turn, difficulties in social relations and the perception of subjective complaints of cognitive functioning are associated with greater personal sense of self-stigma. Finally we consider that these findings may be applied in the clinical management of people with severe mental disorder.

KEY WORDS: Schizophrenia, recovery, self-stigma, metacognition, insight cognitive. 


\section{Introducción}

En los últimos años el abordaje de las personas con patología mental grave se ha orientado hacia el complejo y largo proceso de superación personal llevando consigo el incremento del análisis de los factores intervinientes en el mismo.

El modelo defendido por Andresen et al. (2003) entiende la recuperación como la construcción personal de un proyecto de vida satisfactorio más allá de la evolución de sus síntomas o problemas. Son varios los componentes que intervienen en esta elaboración; el apreciar la esperanza y mantenerla, el restablecimiento de una identidad positiva, el encontrar un sentido a su existencia, y asumir el control sobre la enfermedad y la vida de uno $(1,2)$.

Este trabajo se fundamenta en esta visión que recoge la perspectiva personal y se focaliza en valorar las percepciones subjetivas que subyacen a todo su entramado psicológico.

Corrigan et al. defienden que la recuperación se asocia positivamente con medidas de vida exitosa tales como autoestima, empoderamiento y calidad de vida. También lo relaciona con el soporte social. Indica que tanto la calidad de vida como la recuperación correlacionan inversamente con la sintomatología y con la edad (3).

Entre los factores que inciden en la recuperación personal se encuentra el estigma, tanto en su vertiente social como en su flanco más subjetivo, esto es, el estigma internalizado o autoestigma, aspecto este sobre el que en los últimos años ha aflorado un creciente interés. Se considera al estigma uno de los mayores obstáculos para la recuperación, dado que las consecuencias del autoestigma generan una pérdida de autoestima y autoeficacia que redunda finalmente en reticencias a la interacción (4). En un amplio estudio sobre el estigma en la comunidad de Madrid realizado por Muñoz et al. apreciaron que el aislamiento y el ocultamiento de la enfermedad se utilizan como estrategias ante el rechazo (5).

El grupo que más ha aportado a la investigación sobre el estigma es el liderado por Patrick Corrigan que en distintas publicaciones ha ido proponiendo todo un modelo de funcionamiento del estigma internalizado. El Modelo Social-Cognitivo del Estigma internalizado (6) considera que el estigma internalizado se compone al igual que el estigma público de estereotipos, prejuicios y discriminación, destacando el papel de la identificación con el grupo estigmatizado. Las personas que sufren de enfermedad mental tienen prejuicios contra ellos mismos y tienden a estar de acuerdo con los estereotipos típicos que se asocian a la psicopatología. Explican que esta respuesta personal al estigma de la enfermedad mental se basa en las representaciones colectivas que actúan en esa situación, en la percepción de la legitimidad del estigma, y en la identificación de la persona con el grupo más grande de personas con enfermedad mental. En este contexto, queda patente la gran importancia del soporte social y grupo de referencia inmediato. 
ORIGINALES Y REVISIONES

Livingston y Boyd (7) realizan una de las revisiones internacionales más completas publicada hasta el momento sobre el estigma internalizado detectando que ninguna de las variables sociodemográficas estudiadas; sexo, edad, educación, empleo, estado civil, ingresos económicos y grupo étnico ha demostrado mantener una relación significativa con el estigma internalizado. Sin embargo, hallan que las variables psicosociales tales como esperanza, autoestima, empoderamiento, autoeficacia, calidad de vida, y variables sociales como apoyo social e integración social se relacionan significativamente y generalmente de manera inversa con el autoestigma. En relación a las variables psiquiátricas concluyen que hay una asociación negativa con la adherencia al tratamiento y positiva entre la severidad de los síntomas y el estigma internalizado.

En Europa destaca el estudio GAMIAN-Europa (Global Alliance of Mental Illness Advocacy Networks) (8) que estudia los niveles de autoestigma, de resistencia al estigma y de discriminación percibida. Orientan a que las variables más relacionadas con el estigma internalizado son el autoestima/autoeficacia, empoderamiento, discriminación percibida, conocer y estar de acuerdo con el diagnóstico y un alto número de contactos sociales.

Muñoz et al (9) plantean el Modelo Empírico Socio-Cognitivo Comportamental del estigma internalizado mostrando que las experiencias de discriminación tienen una relación positiva con el estigma internalizado y negativa con las expectativas de recuperación. Encuentran también que las expectativas de recuperación y el estigma internalizado modulan los resultados conductuales, tanto de funcionamiento social como de autonomía personal.

Yanos et al $(10,11)$ exponen un modelo explicativo que profundiza en las relaciones entre el estigma internalizado, la recuperación y los resultados de las intervenciones. A los factores ya mencionados de sintomatología y autoestima incorporan la conciencia de enfermedad, desesperanza y el afrontamiento, apoyando la hipótesis de que el estigma internalizado aumenta los comportamientos evitativos y los síntomas depresivos.

Varios trabajos avalan que la conciencia e interpretación de la enfermedad está relacionada con el autoestigma. La variedad de factores implicados en este proceso va generando una red compleja de interacciones y posibilidades que podría redundar en resultados paradójicos. Plantean que hay resultados contrapuestos respecto al peso de la conciencia de la enfermedad sobre la adherencia, la recuperación y la calidad de vida, y analizan lo que llaman "la paradoja del insight", concluyendo que el insight acompañado de creencias autoestigmatizantes está relacionado con resultados negativos $(12,13)$. El reconocimiento de la enfermedad mental puede ser tanto una clave como un contratiempo para el proceso de la recuperación; es una llave hacia la recuperación en la medida que conduce al cumplimiento terapéutico y consecuente mejora clínica y social, pero la aceptación de la enfermedad 
junto con la aceptación del estigma social asociado puede devenir en detrimento de la recuperación personal por su influencia en mermar la sensación de dignidad personal. Un análisis más matizado realizado por otros autores (14-15) concluye también que el autoestigma modera la asociación entre insight y los resultados, encontrando que los pacientes que tenían alto insight asociado con autoestigma alta tenían más sentimientos de desesperanza y peor funcionamiento social. Por otro lado, estos autores alertan que las personas que tienen un alto insight y alto autoestigma están en riesgo de sufrir experiencias de desmoralización, vivencia contrapuesta a la recuperación. Esta paradoja se resuelve analizando el impacto que tienen los significados que se da a la enfermedad.

En el ámbito psiquiátrico se ha identificado el insight con la capacidad de reconocer que se padece una enfermedad, es decir, con la conciencia de enfermedad. Esta acepción de insight clínico comprende la percepción y aceptación de los trastornos y síntomas, sus consecuencias, la actitud hacia el tratamiento y el cumplimiento. Sin embargo, esta visión del insight no explica los mecanismos que subyacen a la vivencia de estas creencias. Beck et al. (16) proponen el enfoque de insight cognitivo ampliando el tradicional concepto de insight clínico. Ponen el énfasis en el "insight emocional", que implica una suficiente comprensión para modificar creencias disfuncionales y la afectividad asociada así como las consecuencias conductuales de esas creencias, en contraposición al "insight intelectual" que se limita a la adecuada comprensión de los síntomas pero sin experimentar un cambio en el sistema delirante subyacente. En el "insight cognitivo" se acentuaría el mecanismo de evaluación y corrección de interpretaciones inadecuadas y creencias distorsionadas. El análisis de los componentes principales del constructo mostró una estructura de dos factores reconocibles como "autoreflexión", relacionada con la capacidad y disposición a evaluar las propias producciones y la "autocerteza" referida a la sobreconfianza en la validez de las propias creencias.

Riggs et al. (17) emprenden una de las investigaciones que más exhaustivamente estudia el insight cognitivo, tanto en población normal como en subtipos distintos de trastornos, concluyendo que el colectivo afectado con psicosis obtiene de manera significativa menores puntuaciones en autorreflexión e incrementadas en autocerteza respecto al colectivo normalizado.

El funcionamiento cognitivo se encuentra habitualmente afectado en las personas diagnosticadas de esquizofrenia. En esta patología las áreas con mayor deterioro son principalmente la atención, la memoria, la velocidad de procesamiento y el funcionamiento ejecutivo. El deterioro cognitivo es uno de los principales predictores de funcionamiento psicosocial y parece tener una influjo importante en variables relevantes para el tratamiento como el insight y las estrategias de afrontamiento. En este sentido cabe destacar que la valoración subjetiva de los pacientes sobre su propio déficit, es decir, su percepción sobre los síntomas cognitivos 
ORIGINALES Y REVISIONES

constituye una variable relacionada con la calidad de vida y predice el deterioro sintomático. En su Modelo Teórico y Empírico del Insight Cognitivo Beck et al. sugieren una relación directa entre neurocognición e insight cognitivo encontrando que la memoria y el aprendizaje verbal están relacionados con la autocerteza, y la memoria de trabajo y función ejecutiva con la autorreflexión.

Este modelo defiende que las limitaciones cognitivas referidas al déficit en el insight cognitivo, en el que se incluyen las quejas cognitivas, contribuyen al deterioro del insight clínico y al desarrollo de la productividad psicótica, orientado el pronóstico y los resultados en funcionamiento y calidad de vida. Plantean que ambos constructos, insight clínico y cognitivo se complementan. Los pacientes con mayor insight cognitivo son más propensos a vivir de manera independiente y a mantener una mejor calidad de vida, estando estos resultados también asociados con los síntomas.

Este creciente interés en la última década por los aspectos subjetivos incluye el acercamiento a la metacognición. Existe un cierto acuerdo de que se trata de un proceso complejo y tridimensional que abarca la conciencia, monitorización (supervisión, control y regulación) y exploración de los procesos básicos. Las investigaciones sobre el insight clínico y metacognitivo o neuropsicológico son escasos y poco concluyentes, especialmente en lo referente a su relación mutua.

El reconocimiento de la propia afectación cognitiva por parte de las personas con trastornos psicóticos juega un papel muy importante en los déficits residuales observados en las personas afectadas de esquizofrenia por su implicación en la adherencia a intervenciones de rehabilitación cognitiva y contribución en su funcionamiento general. Taste et al. (18) encuentran afinidad entre las quejas neurocognitivas y el insight cognitivo, de tal manera que el reconocimiento de dificultades cognitivas se relacionaba con una mayor tendencia a la autorreflexión y mayor crítica de la ideación extraña. No hay acuerdo en la literatura sobre la capacidad de las personas con trastornos psicóticos para concebir la dimensión de sus problemas cognitivos. Algunas investigaciones concluyen que las valoraciones subjetivas de los pacientes sobre sus habilidades cognitivas no corresponden con la evaluación objetivada mediante pruebas neuropsicológicas (19-23).

Mientras que otros análisis avalan que sí existe autoreconocimiento de sus dificultades cognitivas (24-27). Esta divergencia de resultados podría ser debida a las diferencias metodológicas entre estudios dado que los instrumentos psicométricos empleados son dispares en extensión, exhaustividad e incluso en el contenido de lo analizado. Stip et al. (24) utilizan el SSTICS para valorar esta dimensión subjetiva y concluyen que hay correspondencia entre las quejas subjetivas y los déficits objetivados en el funcionamiento de atención y memoria, constituyendo una dimensión clínica importante del trastorno. Sugieren que los déficits cognitivos subjetivos son prevalentes en este trastorno y que pueden ser valorados de manera fiable. Defien- 
den que las quejas subjetivas son un buen predictor de funcionamiento, adherencia a la medicación, empeoramiento de la sintomatología psicótica y reflejo del estado de bienestar.

Junto a la mejoría de los síntomas, la prevención de recaídas y las discapacidades, la calidad de vida constituye una de las metas fundamentales en cualquier tipo de tratamiento. Aunque su delimitación conceptual no es siempre clara, se identifica más con la valoración subjetiva que la persona hace de su propia vida, en el contexto del sistema cultural en que vive y de sus expectativas e intereses personales

El objetivo primordial de este trabajo es verificar la trascendencia de la percepción del paciente sobre sus procesos cognitivos y su relación con el proceso de recuperación, en especial valorando la preponderancia del autoestigma. Las revisiones aportadas encaminan a esperar que el peso del autoestigma en este proceso sea de gran importancia y de dirección inversa. Esto lleva a comprobar si se dan algunos resultados descritos en la bibliografía tales como explorar la relación entre el funcionamiento cognitivo objetivado y la percepción de cognición subjetiva y el insight cognitivo, la relación entre el insight cognitivo con el autoestigma y la recuperación.

Se contempla el examinar de manera indirecta la relación entre calidad de vida y recuperación. Además de las variables sociodemográficas se tienen también en cuenta aspectos clínicos, funcionales y sociales, que aunque no son nucleares en nuestro examen interesa vislumbrar hacia donde apuntan y cómo quedan afectados.

\section{Material y Método}

Planteamos un diseño analítico descriptivo encaminado a explorar el tipo de asociaciones relacionales entre variables. El corte trasversal del estudio dificulta el proponer resultados concluyentes sobre posibles relaciones causales, aunque permite orientar la importancia predictiva de determinadas variables en el conocimiento del proceso de recuperación.

\section{Sujetos}

El análisis se llevó a cabo en dos hospitales de día de la Red de Salud Mental de Bizkaia del Servicio Vasco de Salud-Osakidetza. La muestra estaba compuesta por 59 personas con diagnóstico de esquizofrenia o trastorno esquizoafectivo que consintieron en participar en el mismo. En la tabla 1 se describe el perfil de la población valorada. 
ORIGINALES Y REVISIONES

\section{Instrumentos}

Las variables sociodemográficas se analizaron mediante la información incluída en las historias clínicas de los casos incorporados.

La valoración del estado global se llevó a cabo con la Escala HoNOS (Health of the Nation Outcome Scales). Se trata de un instrumento de medición desarrollado por la Unidad de Investigación del Royal College of Psychiatrists en el marco del Proyecto Health of the Nation del Departamento de Salud Británico, traducida y validada por Uriarte et al. (28). Está constituido por un conjunto de 12 escalas diseñadas para medir todo el rango general de problemas clínicos y comportamentales, de deterioro y sociales asociados a la enfermedad mental. La máxima puntuación posible es de 48; a mayor puntuación, mayor gravedad.

La exploración neuropsicológica general del deterioro cognitivo objetivado se hizo mediante la Escala Breve para evaluar el Deterioro Cognitivo en Pacientes Psiquiátricos (Screen for Cognitive Impairment in Psychiatriy, SCIP) adaptada y validada (29). El SCIP proporciona una puntuación global de deterioro cognitivo objetivo mediante la evaluación del aprendizaje de palabras inmediato, memoria de trabajo, fluidez verbal, aprendizaje diferido y velocidad de procesamiento.

La satisfacción con la calidad de vida se midió a través de la adaptación del EuroQol-5D, instrumento genérico de medición de la calidad de vida relacionada con la salud, empleándose únicamente la información cuantitativa del bienestar subjetivo global del paciente (0-100).

Para el análisis del funcionamiento autónomo general de los pacientes a lo largo de un continuum de salud-enfermedad se usó la Escala de Evaluación de la Actividad Global (GAF) propuesta por la American Psychiatric Association.

En cuanto a las escalas que evalúan los aspectos subjetivos y sus correspondientes constructos, se han administrado las siguientes:

La valoración subjetiva de los síntomas psicóticos se realizó mediante la Escala Subjetiva para Investigar la Cognición en Esquizofrenia (SSTICS), en su traducción y adaptación de Soriano et al (30). Consta de 21 ítems sobre quejas cognitivas planteadas en el contexto de eventos diarios, divididos en los factores de dificultades relacionadas con la función ejecutiva, la memoria, la conciencia de esfuerzo, la vida diaria, la distracción y la vigilancia.

La metacognición se estudió con la Escala de Metacognición Social para la Psicosis (GEOPTE). Empleamos únicamente la versión autoaplicada del paciente, compuesta de 15 ítems, 7 para funciones metacognitivas básicas y 8 para la metacognición social (31-32).

El autoestigma experimentado se exploró con la adaptación de la Escala de Auto-Estigma (SS) de King realizada por Flores et al (33-34). Es una prueba de 28 ítems con una escala tipo likert que integra la discriminación, divulgación y aspectos positivos de la enfermedad mental. La subescala de discriminación contiene 
reactivos que se refieren a las reacciones negativas de otras personas. La subescala de divulgación indaga sobre la vergüenza o el temor a divulgar o manejar la información acerca de la enfermedad para evitar la discriminación. Finalmente, la subescala de aspectos positivos determina cómo la gente acepta su enfermedad y muestra la forma en que los sujetos se perciben menos afectados por el estigma. A mayor puntuación total, mayor es el nivel de estigma internalizado, pues se invierten los items positivos.

La medición subjetiva de la recuperación se llevó a cabo con la adaptación de la Recovery Assessment Scale (RAS-21), de la escala original de Corrigan et al. $(35,5)$. La escala se aproxima a la apreciación subjetiva de la recuperación a través de 24 items que han resultado del análisis factorial de la escala original que consta de 41 items. Los factores que conforman la escala son la confianza personal y esperanza, predisposición para pedir ayuda, orientación a la meta y al éxito, confianza en otros y no dominación por síntomas.

Para una exploración del insight desde una aproximación cognitiva empleamos la adaptación y validación española de la Escala de Evaluación del Insight Cognitivo de Beck et al. $(16,36)$. Es una medida de autoregistro de 15 ítems que valora cómo los pacientes evalúan su propio juicio. Consta de dos dimensiones, Autoreflexión (9 ítems) y Autocerteza (6 ítems). Se obtiene así mismo un Índice compuesto de Insight Cognitivo como Reflexión-Certeza (IC=R-C) (resta de autocerteza a autoreflexión).

\section{Análisis estadístico}

Los datos se han procesado informáticamente mediante el paquete estadístico SPPSS, versión 20.0. En primer lugar, y con el objetivo de explorar las relaciones entre las variables se han utilizado los estadísticos Chi-cuadrado, t-student, ANOVA y correlaciones bivariadas ( $\mathrm{r}$ de pearson o rho de Spearman) dependiendo de la naturaleza de las variables cuantitativas o cualitativas implicadas en la relación. Posteriormente se han complementado mediante la regresión lineal para analizar los factores asociados con las dos variables de mayor interés, la recuperación y el autoestigma, introduciendo en los modelos como variables predictoras aquéllas que en los análisis univariados habían mostrado alguna relación estadísticamente significativa con las variables criterio (recuperación y autoestigma). 


\section{Resultados}

En la tabla 1 se muestran las características sociodemográficas de la muestra. Los resultados generales de medias y desviaciones típicas estadísticas obtenidas de las pruebas administradas se exponen en la tabla 2. En la tabla 3 se exponen los resultados correlacionales (Pearson) de las puntuaciones totales de las pruebas administradas.

Tabla.1

Variables sociodemográficas

\begin{tabular}{ll} 
Edad Media & 43,64 años \\
\hline Sexo & $80 \%$ hombres \\
\hline Estado civil & $85 \%$ solteros \\
\hline Años de evolución & $90 \%>15$ años \\
\hline Situación legal & $49 \%$ situación de protección jurídica \\
\hline Alojamiento & $44 \%$ alojamiento protegido \\
\hline
\end{tabular}

Tabla 2.

Resultados de las Escalas

Media D.t. Rango

Variables subjetivadas

\begin{tabular}{lccc}
\hline RAS-21. Escala de Recuperación & 83,52 & 0,81 & $24-120$ \\
\hline SS. Escala de autoestigma & 52,54 & 16,32 & $0-112$ \\
\hline SSTICS. Percepción de quejas cognitivas & 26,88 & 15,00 & $0-84$ \\
\hline EICB. Insight Cognitivo. Autorreflexión & 13,29 & 5,32 & $0-27$ \\
\hline EICB. Insight Cognitivo. Autocerteza & 9,02 & 3,97 & $0-18$ \\
\hline EUROQOL. Satisfacción Estado de Salud General & 68,00 & 19,83 & $0-100$ \\
\hline GEOPTE. Metacognición social & 30,05 & 11,17 & $15-75$ \\
\hline
\end{tabular}

Variables objetivadas

\begin{tabular}{lccc} 
GAF. Valoración de la Actividad Global & 47,25 & 7,38 & $0-90$ \\
\hline SCIP. Screening cognitivo general & 59,64 & 13,08 & \\
\hline HoNos Total. Estado general & 9,05 & 3,56 & $0-48$ \\
\hline
\end{tabular}


Tabla3.

Resultados correlacionales (pearson) entre las pruebas administradas

\begin{tabular}{lcccccccccccc}
\hline & $\mathbf{1}$ & $\mathbf{2}$ & $\mathbf{3}$ & $\mathbf{4}$ & $\mathbf{5}$ & $\mathbf{6}$ & $\mathbf{7}$ & $\mathbf{8}$ & $\mathbf{9}$ & $\mathbf{1 0}$ & $\mathbf{1 1}$ & $\mathbf{1 2}$ \\
\hline 1. HONOS item 3 & 1 & & & & & & & & & & & \\
2. HONOS item 6 &, 100 & 1 & & & & & & & & & \\
3. HONOS item 9 &,- 082 &, 164 & 1 & & & & & & & & \\
4. PT SCIP &,- 116 &,- 025 &, 021 & 1 & & & & & & & \\
5. PT GAF &,- 211 &,- 185 &,- 044 &,- 154 & 1 & & & & & & \\
6. PT SSTICS &,- 234 &, 015 &, 082 &, 021 &, 034 & 1 & & & & & \\
7. PT GEOPTE &,- 111 &,- 096 &, $266^{*}$ &, 080 &,- 020 &, $647^{* *}$ & 1 & & & & \\
8. P. EICB Autorreflexión &,$- 280^{*}$ &,- 073 &,- 072 &,- 135 &, 237 &, $289^{*}$ &, $276^{*}$ & 1 & & & \\
9. P. EICB Autocerteza &,- 054 & -254 &, 162 &,- 008 &, 035 &,- 104 &,- 193 &, 143 & 1 & & \\
10. PT SS King &,- 039 &,- 121 &, $281^{*}$ &, 144 &,- 088 &, $406^{* *}$ &, $421^{* *}$ &, 162 &,- 146 & 1 & & \\
11. PT RAS &, 117 &,$- 327^{*}$ &,$- 331^{*}$ &,- 202 &,- 019 &,- 193 &,- 221 &,- 137 &,- 014 &,$- 367^{* *}$ & 1 & \\
12. PT Estado Hoy &,- 083 &, 220 &, 073 &,- 041 &, 039 &,$- 337^{* *}$ &,$- 275^{*}$ &,- 025 &, 092 &,$- 348^{* *}$ &, 100 & 1 \\
\hline
\end{tabular}

*. La correlación es significante al nivel 0,05 (bilateral).

**. La correlación es significativa al nivel 0,01 (bilateral).

\section{Variables sociodemográficas}

Recabados los resultados sociodemográficos encontramos que el sexo no se relaciona especialmente con ninguna variable a excepción del estado civil, siendo significativo el estatus de casada en las mujeres frente a los hombres, (rho $=-0,402 ; \mathrm{p}<0,05)$.

En relación a las variables cronológicas, encontramos que la edad tiene una relación inversa con las puntuaciones en la escala GAF $(r=-0,316 ; \mathrm{p}<0,05)$ y la de autorreflexión $(\mathrm{r}=-0,317 ; \mathrm{p}<0,05)$, y una relación inversa con mayores puntuaciones en el SCIP $(r=-0,443 ; \mathrm{p}<0,01)$. Las puntuaciones de la escala GAF se relacionan inversamente con la protección jurídica $(\mathrm{rho}=-0,501 ; \mathrm{p}<0,01)$ y el soporte de alojamiento $(\mathrm{rho}=-0,300 ; \mathrm{p}<0,05)$.

Relación entre el funcionamiento cognitivo objetivo y la metacognición.

En lo que atañe a la exploración del déficit cognitivo global no aparece relación entre los resultados de la SCIP con la SSTICS, ni con la GEOPTE. Sin embargo sí se muestra que subpruebas específicas del SCIP como la mejor fluidez $(\mathrm{r}=0,299 ; \mathrm{p}<0,05)$ y habilidad visomotora $(\mathrm{r}=0,259 ; \mathrm{p}<0,05)$ están relacionadas con una mejor tendencia a la autorreflexión.

Las puntuaciones en la escala GEOPTE indican relaciones significativas de dirección positiva con la escala SSTICS y la escala SS, y negativas con la EUROQOL de tal manera que la mayor conciencia sobre las dificultades cognitivas propias y de interacción social, sale vinculada a la presencia de estigma internalizado y menor satisfacción con el estado de salud. De igual modo, los valores obtenidos en la escala SSTICS se relacionan inversamente con los obtenidos en la EUROQOL. 
Respecto a las puntuaciones en la escala EICB destaca la relación significativa de la subescala autorreflexión con el resto de variables subjetivas relacionadas con el examen del propio funcionamiento intelectivo, como el factor neurocognitivo de la escala GEOPTE y las quejas cognitivas de la escala SSTICS. No aparece relación con la puntuación global de la escala SS pero su subfactor de percepción de discriminación se relaciona positivamente con la autorreflexión $(\mathrm{r}=0,285$; $\mathrm{p}<0,05)$.

Respecto a las áreas que mide la HoNOS y su relación con las variables cognitivas, se aprecia que el consumo de tóxicos (ítem 3 de la escala HoNOS) se relaciona inversamente con la puntuación en autorreflexión, mientras que el ítem 9 , referente a las relaciones sociales, se asocia significativa y positivamente con las puntuaciones en la escala GEOPTE.

Variables asociadas con la experiencia de recuperación y con el autoestigma

En los análisis univariados se pone de manifiesto que una menor puntuación en la escala RAS se asocia con puntuaciones más altas en la escala de autoestigma y con mayores puntuaciones en las áreas 6 y 9 de la HoNOS que hacen referencia a los problemas derivados de la presencia de delirios y alucinaciones y a los problemas en las relaciones sociales. Además, las puntuaciones en la escala RAS de recuperación se relacionan inversamente con el subfactor de presencia de déficit neurocognitivo de la escala GEOPTE $(r=-0,280 ; p<0,05)$. No se aprecia relación entre los resultados de la escala RAS y la EICB de insight cognitivo.

Por su parte, las puntuaciones en la escala SS que valora autoestigma aparecen vinculadas directamente con la escala SSTICS y GEOPTE, con significaciones a nivel de $p<0,01$. Mayores puntuaciones en la escala de autoestigma se asocian también a mayores problemas interpersonales de acuerdo a la valoración en el ítem 9 de la HoNOS, encontrándose una relación inversa con la puntuación obtenida en la escala de Calidad de Vida.

Un análisis más exhaustivo a través de la regresión lineal señala dos factores que se asocian con mayores puntuaciones en la escala SS, es decir, con un mayor autoestigma: la puntuación en la escala SSTICS, percepción de dificultades cognitivas $(\beta=0,385 ; \mathrm{T}=3,269 ; \mathrm{p}<0,02)$ y el ítem 9 de la escala HONOS, los problemas en las relaciones sociales $(\beta=0,25 ; \mathrm{T}=2,119 ; \mathrm{p}<0,03)$, que explican juntas el $22,7 \%$ de la varianza del autoestigma.

Cuando se considera como variable criterio la puntuación en la escala de recuperación, la prueba de regresión lineal denota como variables predictivas al autoestigma $(\beta=-0,412 ; \mathrm{T}=-4,72 ; \mathrm{p}<0,01)$ y la presencia de sintomatología psicótica $(\beta=-0,376 ; \mathrm{T}=-3,597 ; \mathrm{p}<0,02)$, explicando el $27,4 \%$ de la varianza y mostrando que a mayor sintomatología productiva y estigma autopercibido, menor es la vivencia de recuperación. 


\section{Discusion}

Analizados todos los datos, los hallazgos más sobresalientes destacan entorno a la variable recuperación y la variable autoestigma, siendo estas, junto a la variable metacognición social, las que más interacciones relacionales establecen.

La interpretación de los resultados apunta a que el proceso de recuperación personal queda negativamente influenciado por la sensación de autoestigma y presencia de actividad productiva, estando también asociado con las dificultades relacionales y con aspectos subjetivos tales como la percepción y atribución que se hacen de las dificultades cognitivas.

Se desprende que el autoestigma queda afectado fundamentalmente por la calidad de las relaciones sociales y por la sensación de tener dificultades cognitivas, lo que concuerda con las conclusiones planteadas en el estudio GAMIAN (8) y de Muñoz et al. (9). Se relaciona también con los problemas en metacognición social y con la escasa satisfacción con el estado general de salud. Destacar que las puntuaciones obtenidas en autoestigma son inferiores a las citadas en la literatura. Dada la escasez de referencias sobre resultados estratificados nos dificulta contrastar, establecer relaciones y justificar determinados hallazgos.

No encontramos respaldo para relacionar ni el autoestigma y ni la recuperación con la autonomía personal objetivada. En este último sentido merecería la pena utilizar pruebas de valoración funcional más específicas y mediciones que contrasten y recojan la percepción de los pacientes sobre esta área.

Deteniéndonos en el rendimiento cognitivo objetivo, no se encuentra concordancia entre lo referido subjetivamente por los usuarios y su rendimiento en pruebas objetivas estandarizadas. Unicamente se apunta que a mejor rendimiento en las tareas que evalúan flexibilidad cognitiva y velocidad de procesamiento, mayor es la habilidad de autorreflexión. Por tanto, se obtienen resultados similares a los referidos en la introducción (19-23). Estos desencuentros pueden ser explicados por varias razones; pudiera ser que el tamaño muestral y la especificidad del colectivo cribado; con problemática abigarrada y multivariada, muchos años de evolución, pueda estar influyendo en la ausencia de hallazgos en esta dirección. También esta circunstancia condiciona la extrapolación de estos resultados.

Podemos atribuirlo también a condicionantes metodológicos ya apuntados en relación a los escasos estudios sobre las variables cognitivas subjetivas hechos con pruebas heterogéneas, y también atribuibles a la disparidad en las pruebas de detección del déficit objetivo. De hecho las pruebas de valoración neuropsicológica elegidas simplemente cubren un tanteo general del estado cognitivo por lo que no son sensibles ni muy específicas. Sería preciso establecer una batería de pruebas cognitivas más exhaustiva y diversa para profundizar en este sentido. Como tendencia de observación también se puede tener en cuenta que una de las áreas que 
ORIGINALES Y REVISIONES

no valora el SCIP es la función ejecutiva, aspecto ampliamente relacionado con el insight y por tanto, no solo con resultados funcionales sino también con subjetivos. No aparece ninguna relación significativa entre autocerteza y las funciones cognitivas valoradas por el SCIP, ni tampoco entre esta última y la metacognición social. Ello sugiere la existencia de otras variables moduladoras que estarían interviniendo en la percepción de la cognición social. Podría explicarse por un mecanismo de adaptación a su situación cotidiana ritualizada en el que se suplen y compensan o no precisan de habilidades cognitivas que aparecen deficitarias en las pruebas cognitivas que redundaría en no experimentar carencia de las mismas. Por tanto, se hace fundamental entender estos resultados de una manera ecológica y contextual, y explorar también la sensación subjetiva de autonomía personal.

Para algunos investigadores estos resultados discordantes están ligados al hecho de que los sujetos de este colectivo presentan problemas relacionados con la conciencia de enfermedad o insight. En este análisis el insight clínico no ha sido valorado por lo que esta atribución no es defendible, pero sí se ha tenido en cuenta la elaboración que realizan sobre su propio pensamiento. En primer lugar, resulta relevante que sí se encuentra concordancia interna entre todas las pruebas que evalúan dimensiones subjetivas como metacognición básica, metacognición social, autorreflexión y quejas cognitivas. Es importante tener en cuenta este dato, ya que nos muestra que los resultados encontrados no son un artefacto metodológico sino que son un reflejo de una autopercepción coherente y estable. Queda respaldada la relación del insight cognitivo con el resto de variables autopercibidas, acordando con lo planteado inicialmente y avalando la consistencia de tales impresiones (18). A pesar de que parece que la sensación subjetiva respecto al rendimiento cognitivo es independiente de los resultados objetivos, hay asociación entre la alteración objetiva de las interacciones relacionales y la percepción de deterioro de las capacidades cognitivas sociales en ellas implicadas, pues la mayor dificultad relacional apreciada desde el juicio clínico y la deficitaria metacognición social salen vinculadas directamente.

Siguiendo los datos aportados se comprueba la gran relevancia que en este análisis tiene la relación social. La importancia moduladora de las relaciones sociales ha aparecido altamente relacionada con el autoestigma, especialmente con la sensación de discriminación, apuntando a lo que plantea las tesis de Corrigan. En el planteamiento inicial de este estudio no se consideraba la variable social como un aspecto nodal y por ello no se valoró más que a través de la impresión clínica desde el ítem 9 de la escala HoNOs. Dada la trascendencia de este factor y los resultados obtenidos merecería tenerse en cuenta en posteriores investigaciones de una manera más central y valorada por pruebas más específicas, tanto objetivas como subjetivas.

No se detecta asociación entre la satisfacción con el estado de salud y la recuperación, sin embargo el bienestar percibido sí se relaciona con la ausencia de 
quejas subjetivas cognitivas y de deterioro social, y con la falta de estigma internalizado. Esto último está en consonancia con los resultados de Livinston y Boyd (7).

\section{Consideraciones para la práctica clínica}

Los resultados denotan la importancia crucial que tiene la percepción y atribuciones que realizan los pacientes sobre sus dificultades cognitivas como elementos clave para afectar a la internalización de determinadas ideas, sentirse estigmatizados y consecuentemente entorpecer su proceso de recuperación. Esto deriva en que, tal como lo defienden varios autores citados $(10,26)$, será preciso priorizar el abordaje de estos aspectos en la práctica clínica. Tal como defiende Kurvic et al. (37), la baja orientación a la recuperación y el autoestigma incrementado podrían socavar la alianza terapéutica independientemente del efecto perjudicial del pobre insight clínico, comprometiendo cualquier desarrollo terapéutico.

En la mayoría de las entrevistas estructuradas asistenciales se obvia la valoración de la experiencia subjetiva, lo cual es preciso reconsiderar dado el ascendente que las variables subjetivas tienen como expresión de vivencias personales y como facilitadoras de la implicación en el proceso terapéutico.

Es fundamental atender las variables subjetivas cognitivas relacionadas con la cognición social, el insight y el estigma internalizado para ayudar a encaminar el proceso de recuperación de una persona. Se considera que el insight cognitivo es un buen marcador para el pronóstico porque alude a la capacidad de reflexionar, pudiendo ser un indicador del insight emocional del que nos habla Beck, salvando la disociación entre lo que se aprende a contestar en relación con la enfermedad y lo que se siente realmente.

El proceso de rehabilitación psicosocial deberá contemplar propulsar la red social de la persona como proveedora de grupo identitario de referencia y trasmisora de habilitación y cultura libre de estigmatización, con objeto de mejorar la autoestima, la autoeficacia y la calidad de vida. Esto atañe también al ámbito profesional en el que se desenvuelve, máxime cuando para muchas personas con enfermedad mental grave de larga evolución este suele ser el medio relacional con el que más interaccionan. Es por ello que se hace necesario revisar el nivel de orientación de los servicios hacia la recuperación y la actitud de los profesionales.

Quedan significadas algunas de las facetas sobre las que es preciso focalizar la atención terapéutica con objeto de capacitar y contribuir a la superación del autoestigma para promover el camino hacia la recuperación. 
ORIGINALES Y REVISIONES

\section{BIBLIOGRAFÍA}

(1) Andresen, R., Oades, L., \& Caputi. The experience of recovery from schizophrenia: towards an empirically validated stage model. Australian and New Zealand Journal of Psychiatry, $2003 ; 37,586-594$.

(2) Shepherd G, Boardman J.\& Slade M. Haciendo de la recuperación una realidad. Sainsbury Centre for Mental Health, 2008.

(3) Corrigan P.W. Recovery as a Psychological Construct. Community Mental Health Journal, 1999; Vol. 35, No. 3. 231-240.

(4) Manrique Gálvez E., Bravo Alba F. Estigma y cognición social ; estrategias para promover el cambio personal y social. Revista de Psiquiatría y Salud Mental. Hermilio Valdizán, JulioDiciembre 2004; Vol. V. N², pp 3-16.

(5) Muñoz M., Pérez-Sants E., Crespo M., Guillén A.E. Estigma y enfermedad mental. Editorial complutense, 2009.

(6) Corrigan P.W., Watson A. The Paradox of Self-Stigma and Mental Illness. Clinical Psychology: Science and Practice, March 2002; Volume 9, Issue 1, pages 35-53.

(7) Livingston, J. D., \& Boyd, J. E. (2010). Correlates and consequences of internalizedstigma for people living with mental illness: A systematic review and meta-analysis.Social Science \& Medicine, 71,2150-2161. doi: 10.1016/j.socscimed.2010.09.030.

(8) Brohan E., Elgie R., Sartorius N., Thornicroft G. Self-stigma, empowerment and perceived discrimination among people with schizophrenia in 14 European countries: The GAMIAN-Europe study. Schizophr Res, 2010; Sep;122(1-3):232-8. doi: 10.1016/j.schres. 2010.02.1065.

(9) Muñoz M., Sanz M., Perez-Santos E. Estado del conocimiento del estigma internalizado desde el modelo socio-cognitivo -comportamental. Anuario de Psicología Clínica y de la Salud, 2011, Vol 07, pag. 41-50.

(10) Yanos P.T., Roe D., Markus K., Lysaker P.H. Pathways Between Internalized Stigma and Outcomes Related to Recovery in Schizophrenia Spectrum Disorders. Psychiatric Services, 2008; 59(12). 1437-1442. doi: 10.1176/appi.ps.59.12.1437.

(11) Lysaker.P.H., Olesek K.L., Warman D.M. , Martin J.M., SalzmanA.K., Nicolò G., Salvatore G., Dimaggio G. Metacognition in schizophrenia: Correlates and stability of deficits in theory of mind and self-reflectivity. Psychiatry Res. 2011; 190 (1),18-22 doi:10.1016/j.psychres.2010.07.016.

(12) Lysaker P.H, Roe D., and Yanos P.T. Toward Understanding the Insight Paradox: Internalized Stigma Moderates the Association Between Insight and Social Functioning, Hope, and Selfesteem Among People with Schizophrenia Spectrum Disorders. Schizophrenia Bulletin,2007; vol. 33 no. 1 pp. 192-199, 2007 doi:10.1093/schbul/sbl016 Advance Access.

(13) Lysaker P.H., Buck K.D. Insight, Outcome and Recovery in Schizophrenia Spectrum Disorders: An Examination of their Paradoxical Relationship. Current Psychiatry Reviews, 2007; 3, 65-71.

(14) Staring AB., Van der Gaag M., Van den Berge M., Duivenvoorden H.J., Mulder C.L. Stigma moderates the associations of insight with depressed mood, low self-esteem, and low quality of life in patients with schizophrenia spectrum disorders. Schizophrenia Research, 2009; 115 (2009) 363-369.

(15) Calvelti M., Kvrgic S., Beck E., Rüsch N., Vauth R. Self-stigma its relationship with insight, demoralization, and clinical outcome among people with schizophrenia spectrum disorders. 2012; Comprensive Psychiatry 53, (2012) 468-479. 
(16) Beck AT., Baruch E., Balter JM., Steer RA., Warman DM. A new instrument for measuring insight: the Beck Cognitive Insight Scale. Schizophr Res. 2004; 68(2-3):319-29.

(17) Riggs SE., Grant PM., Perivoliotis D., Beck AT. Assessment of Cognitive Insight: A cualitative review. Schizophr Bull. 2012; 38(2):338-50. doi: 10.1093/schbul/sbq085.

(18) Tastet H., Verdoux H., Bergua V., Destaillats JM., Prouteau. A. Cognitive insight in schizophrenia. The missing link between insight and neurocognitive complaint? The Journal of Nervous and Mental Disease .2012; J. Nerv. Ment. Dis. 200 (10): 908-10.

(19) Medalia A., Lim R.W. Self-awareness of cognitive functioning in schizophrenia. Schizophrenia Research, 2004; 71: 331-338.

(20) Medalia A., Thysen J.A. Freilich B. Do people with schizophrenia who have objective cognitive impairment identify cognitive deficits on a self report measure? Schizophrenia Research, 2008; 105: 156-164.

(21) Medalia A., Thysen J.A. Comparison of insight into clinical symptoms versus insight into neuro-cognitive symptoms in schizophrenia. Schizophrenia Research, 2010; 118: 134-139.

(22) Chan RCK., Wang Y., Ma Z, Hong XH., Yuan Y., Yu X., et al. Objective measures of prospective memory do not correlate with subjective complaints in schizophrenia. Schizophr Res, 2008; 103:229-39.

(23) Johnson I., Tabbane K., Dellagi L., Kebir O. Self-perceived cognitive functioning does not correlate with objective measures of cognition in schizophrenia. Comprehensive Psychiatry, $2011 ; 52,688-692$.

(24) Stip E., Caron J., Renaud S., Pampoulova T., lecomte Y. Exploring cognitive complaints in schizophrenia: the subjective scale to investigate cognition in schizophrenia. Comprehensive Psychiatry, 2003; 44: 331-340.

(25) Lecardeur L., Briand C., Prouteau A., Lalonde P., Nicole L., Lesage A., Stip E. Preserved awareness of their cognitive deficits in patients with schizophrenia: convergent validity of the SSTICS. Schizophrenia Research, 2009; 107: 303-306.

(26) Bengoechea R., Gil D, Fernández M., Arrieta M., Sánchez R., Prat R., Arce A., Álvarez A. Percepción subjetiva de déficit cognitivos en esquizofrenia. Su relación con insight y otras medidas cognitivas. Revista de Psiquiatría y Salud Mental, 2010; 3 (2): 55-60.

(27) Sanjuán J., Aguilar EJ., Olivares JM., Ros S., Montejo AL., Mayoral F., Gonzalez-Torres MA., Bousoño M. Subjective perception of cognitive deficit in psychotic patients. J Nerv Ment Dis. 2006 Jan;194(1):58-60.

(28) Uriarte, J.J., Beramendi, V., Medrano, J., Wing, J.K., Beevor, A.S. y Curtis, R. Presentación de la traducción al castellano de la Escala HoNOS (Health of the Nation Outcome Scales). Psiquiatría Pública, 1999; 11, 93-101.

(29) Pino, O., Guilera, G., Gómez, J., Rojo, E., Vallejo, J., Purdon, S.E. Escala breve para evaluar la afectación cognitiva en pacientes psiquiátricos. Psicothema, 2006; 18 (3), 447-452.

(30) Soriano MF., Jiménez JF. Escala Subjetiva para investigar la Cognición en la Esquizofrenia. Portal de Salud Mental, 2010; Disponible en: http://portalsaludmental.com/pdf/Evaluacion/ Escala cognitiva subjetiva.pdf.

(31) Sanjuan J., Prieto L., Olivares JM., Ros S., Montejo A., Ferrer F., Mayoral F., GonzálezTorres MA., Bousono M. GEOPTE Scale of social cognition for psychosis. Actas Esp Psiquiatr, 2003; $31: 120-128$. 
ORIGINALES Y REVISIONES

(32) Bosuño M., Gonzalez Torres MA., Montejo A., Mayoral F.,Olivares JM.; Ros S, Sanjuan J. (Grupo GEOPTE). Guía GEOPTE. Ed. Lilly. 2005.

(33) King M., Dinos S., Shaw J.,Watson R., Stevens S., Passetti F., Weich S., Serfaty M. The stigma scale: development of a standardized measure of the stigma of mental illness. Br J Psychiatry, 2007; 190: 248-254. doi : 10.112 / bjp. bp.10 6.024638 .

(34) Flores Reynoso S., Medina Dávalos R.,Robles García R. Estudio de traducción al español y evaluación psicométrica de una escala para medir el estigma internalizado en pacientes con trastornos mentales graves. Salud Mental, 2011; 34: 333-339.

(35) Corrigan P.W., Salver M., Ralph R.O., Songster Y., and Keck L. Examining the Factor Structure of the Recovery Assessment Scale. Schizophrenia Bulletin, 2004; Vol. 30, No. 4. 10351041.

(36) Gutiérrez-Zotes J.A., Valero J., Cortés M.A., Labad A., Ochoa S., Ahuir M., Carlson, J., Bernardo M., Cañizares S., Escarti G., Cañete J., Gallo, Salamero M. Adaptación española de la Escala de Insight Cognitivo de Beck (EICB) en esquizofrénicos. Actas Esp Psiquiatr, 2012; 40(1):2-9.

(37) Kvrgic S., Cavelti M., Beck M., Rusch N., Vauth R. Therapeutic Alliance in schizophrenia: The role of recovery orientation, self-stigma, and insight. Psychiatry Research, 2013, 209(1):15-20. 